Journal of

PERIODONTAL RESEAR CH

\title{
Strontium enhances proliferation and osteogenic behavior of periodontal ligament cells in vitro
}

\begin{tabular}{|r|l|}
\hline Journal: & Journal of Periodontal Research \\
\hline Manuscript ID & JRE-11-17-4308.R3 \\
\hline Manuscript Type: & Original Article \\
\hline Date Submitted by the Author: & n/a \\
\hline Complete List of Authors: & $\begin{array}{l}\text { Bizelli-Silveira, Carolina; Aarhus Universitet Health, Dentistry and Oral } \\
\text { Health } \\
\text { Pullisaar, Helen; Aarhus Universitet Health, Dentistry and Oral Health } \\
\text { Abildtrup, Lisbeth; Aarhus Universitet Health, Dentistry and Oral Health } \\
\text { Andersen, Ole; Interdisciplinary Nanoscience Center (iNANO) } \\
\text { Spin-Neto, Rubens; Aarhus Universitet Health, Dentistry and Oral Health } \\
\text { Foss, Morten; Interdisciplinary Nanoscience Center (iNANO) } \\
\text { Kraft, David ; Aarhus Universitet Health, Dentistry and Oral Health }\end{array}$ \\
\hline Keywords: & \begin{tabular}{l} 
Periodontal ligament, In vitro model, Osteoblast, Regeneration \\
\hline
\end{tabular} \\
\hline
\end{tabular}

\section{SCHOLARONE"}




\section{Strontium enhances proliferation and osteogenic behavior of periodontal ligament} cells in vitro

C. Bizelli-Silveira ${ }^{1,2}$, H. Pullisaar ${ }^{1}$, L.A. Abildtrup ${ }^{1}$, O.Z. Andersen ${ }^{2}$, R. Spin-Neto ${ }^{1}$, M. Foss $^{2}$, D.C.E. Kraft ${ }^{1}$

${ }^{1}$ Department of Dentistry and Oral Health, Faculty of Health, Aarhus University, Aarhus, Denmark.

${ }^{2}$ Department of Physics and Astronomy, Interdisciplinary Nanoscience Center (iNANO), Faculty of Science and Technology, Aarhus University, Aarhus, Denmark.

\section{Corresponding author:}

Carolina Bizelli-Silveira

Department of Dentistry and Oral Health - Aarhus University

Vennelyst Boulevard 9

DK-8000 Aarhus C, Denmark

Phone: +45 42434896

E-mail: cbs@dent.au.dk

Key words: Strontium; cell differentiation; osteogenic; periodontal ligament 


\title{
Strontium enhances proliferation and osteogenic behavior of periodontal ligament cells in vitro
}

\begin{abstract}
Background and Objective: Strontium (Sr) enhances osteogenic differentiation of certain multipotent cells. Periodontal ligament cells (PDLCs) are known to be multipotent, and $\mathrm{Sr}$ might be useful in periodontal bone-tissue engineering. This study investigates the effect of high concentration of $\mathrm{Sr}$ on the proliferation and osteogenic behavior of PDLCs in vitro. Material and Methods: Primary human PDLCs were cultured in MEM $+10 \%$ FBS without (Ctrl) or with $\mathrm{Sr}$ in four diverse concentrations: $\mathrm{Sr} 1,11.3 \times 10^{-3} \mathrm{mg} / \mathrm{L}$, human serum physiological level; Sr2, $13 \mathrm{mg} / \mathrm{L}$, typical human serum level after strontium ranelate treatment; Sr3, $130 \mathrm{mg} / \mathrm{L}$, and Sr4, $360 \mathrm{mg} / \mathrm{L}$. The spreading area (2, 4, 6, 24 hours), proliferation rate (1, 3, 7 days), osteogenic behavior (alkaline phosphatase - ALP activity, 7 and 14 days; expression of osteogenic genes, ALP, Runt-related transcription factor 2 RUNX2, osteopontin - OPN, osteocalcin - OCN, and osteoprotegerin - OPG, 1, 3, 7, 14, 21 days), and formation of mineralized nodules (14 and 21 days) of the PDLCs were assessed. Data was compared group- and period-wise using ANOVA tests.

Results: PDLCs cultured with Sr4 showed increased spreading area (after 4 hours), proliferation rate (from 3 days), and OCN and OPN (from 7 days) gene expression as compared to $\mathrm{Ctrl}, \mathrm{Sr} 1, \mathrm{Sr}$, and $\mathrm{Sr} 3$. Sr4 also led to lower ALP activity (from 7 days), ALP (from 3 days) and RUNX2 (at 7 and 14 days) gene expression, together with more evident formation of mineralized nodules, compared to $\mathrm{Ctrl}, \mathrm{Sr} 1, \mathrm{Sr} 2$, and $\mathrm{Sr} 3$.

Conclusion: PDLCs responded to $\mathrm{Sr} 4$ with increased cellular proliferation and osteogenic behavior in vitro.
\end{abstract}

Key words: Strontium; cell differentiation; osteogenic; periodontal ligament 


\section{Introduction}

Periodontitis is an inflammatory disease that progressively damages the periodontium, including alveolar bone, periodontal ligament (PDL), and root cementum. ${ }^{1,2}$ The desire to develop more favorable treatment options to restore the periodontal structure and regain its physiological function has inspired advanced research into periodontal tissue engineering. ${ }^{2}$ Regenerative periodontal therapy can be used to improve the short- and long-term clinical outcomes of periodontally compromised teeth presenting with deep pockets and reduced periodontal support. ${ }^{3}$ The management of periodontal defects is mainly a result of the collective treatment of the three unique and very specialized periodontal tissues: the PDL, the root cementum, and the alveolar bone. ${ }^{1,3}$

Periodontal regeneration is an extremely complex process that requires new bone, cementum, and connective tissue formation. ${ }^{1}$ PDL cells (PDLCs) present multilineage differentiation capacity, playing a key role in periodontal regeneration. ${ }^{4,5}$ The multipotent mesenchymal stem cells contained in the PDL tissue are essential for the ongoing remodelling of periodontium, as well as for the healing response to injury. ${ }^{6} \mathrm{~A}$ recent study suggests that approximately $30 \%$ of a population of PDLCs possess replicative and multipotency potential, and thus could be steered to differentiate into cells with osteoblastand cementoblast-like properties. ${ }^{7}$ The outcome of such regenerative potential include the capacity to form mineralised nodules, expression of the bone- and cementum-associated markers, and response to bone inductive factors. $4,5,7,8$

Agents stimulating proliferation and differentiation of PDLCs into osteoblast-like cells are of interest for periodontal tissue regeneration. ${ }^{9}$ Such agents could lead to enhanced tissue regeneration by guiding the PDLC population to produce lost bone tissue. ${ }^{7}$ Regarding the differentiation of PDLCs into osteoblast-like cells, the use of strontium (Sr) as a stimulating agent is a feasible possibility. ${ }^{10,11} \mathrm{Sr}$ was first advocated as an adjunct for the treatment of osteoporosis, and has been suggested to perform a dual action in bone 
tissue, both stimulating bone formation and suppressing bone resorption, simultaneously. ${ }^{12}$ Studies on the effect of systemic treatment with Sr have suggested that this element might be an effective route to enhance the regeneration of bone defects, and the osseointegration of titanium implants. ${ }^{13,14}$ Based on the osteogenic activity induced by Sr, the use of this element in periodontal regeneration would be reasonable. However, only few studies have addressed the direct interaction between $\mathrm{Sr}$ and the behaviour of PDLCs. ${ }^{15,16}$ One of these studies suggests non-toxic effects of $\mathrm{Sr}$, in the form of strontium ranelate, when used in concentrations as high as $20 \mathrm{mg} / \mathrm{mL},{ }^{15}$ while the other ratifies its possibility to promote PDLCs proliferation, at a concentration of $3 \mathrm{mM}$, or approximately $260 \mathrm{mg} / \mathrm{L}$ (in the form of $\mathrm{SrCl}_{2}$ ). ${ }^{16}$ The effects of varying concentrations of $\mathrm{Sr}$ on the osteogenic behavior of PDLCs, and the proper genetic pathways, which are activated when this stimulating agent is used, have not been investigated.

Thus, the objective of the present study was to investigate the effect of a high concentration of Sr on PDLCs growth and osteogenic behavior.

\section{Material and methods}

Primary cell culture

Human PDLCs were isolated from an impacted fully developed healthy third molar, with an indication for extraction, which was surgically removed from a 19-year-old female patient. Informed consent was obtained and the PDLCs were collected and used in this study. Following surgical removal of the third molar, PDL tissue from the middle third of the tooth roots was scraped with a scalpel under sterile conditions. The PDL tissue was enzymatically digested for 30 minutes at $37^{\circ} \mathrm{C}$ in minimum essential medium (MEM; Sigma-Aldrich, St. Louis, USA) containing $3 \mathrm{mg} / \mathrm{mL}$ collagenase type I (Medinova, Zürich, Switzerland) and 2.4 units/mL dispase II (Roche Diagnostics, Mannheim, Germany). Single PDLC suspensions were obtained by filtration through $70 \mu \mathrm{m}$ cell sieves. The 
isolated PDLCs were cultured in MEM supplemented with 10\% fetal bovine serum (SigmaAldrich, St. Louis, USA) and antibiotics $(25.000 \mathrm{IU} / \mathrm{mL}$ penicillin and $25 \mathrm{mg} / \mathrm{mL}$ streptomycin; DuraScan Medical Products, Odense, Denmark) at $37^{\circ} \mathrm{C}$ in an atmosphere of $100 \%$ relative humidity and $5 \% \mathrm{CO}_{2}$. Cell experiments (cell morphology assessment, cell proliferation analysis, osteogenic behavior assessment, and mineralization analysis) were undertaken with PDLCs in the third passage, following the evaluation periods selected for each of the tests.

\section{Strontium concentration}

Cells were cultured without (Ctrl) or with one of four increasing Sr concentrations, defining the group they were allocated to: $\mathrm{Sr} 1$, the physiological level of $\mathrm{Sr}$ in the human serum $\left(11.3 \times 10^{-3} \mathrm{mg} / \mathrm{L}\right), \mathrm{Sr} 2$ (human serum concentration reached after culture with a common Sr systemic supplementation used by osteoporotic women, $13 \mathrm{mg} / \mathrm{L}) ; \mathrm{Sr} 3$, ten times the $\mathrm{Sr} 2$ concentration $(130 \mathrm{mg} / \mathrm{L})$, and $\mathrm{Sr} 4$, approximately thirty times the $\mathrm{Sr} 2$ concentration (360 $\mathrm{mg} / \mathrm{L}$, a higher concentration than that previously tested in the literature). Strontium was added as strontium chloride hexahydrate $\left(\mathrm{SrCl}_{2} \cdot 6 \mathrm{H}_{2} \mathrm{O}\right.$, Sigma-Aldrich, St. Louis, USA).

\section{Cell morphology - spreading area}

Cells were seeded at a density of $15 \times 10^{3} \mathrm{cells} / \mathrm{cm}^{2}$ in 24-well plates (containing a round regular plastic coverslip - Sigma-Aldrich, St. Louis, USA - $\varnothing 12 \mathrm{~mm}$ - in the bottom of the well), divided according to the groups (Ctrl and $\mathrm{Sr} 1-4)$. The cells were fixed with $10 \%$ formalin solution (approximately 4\% formaldehyde) after 2, 4, 6, and 24 hours of culture. Quantification of cell spreading area was carried out using immunofluorescence. For cell counting, the nucleus was stained using DAPI nucleic acid stain (D9542, Sigma-Aldrich, St. Louis, USA). The F-action in the cytoskeleton and vinculin protein were stained with 
Phalloidin (P1951, Sigma-Aldrich, St. Louis, USA) and Alexa Fluor 488 (Goat anti-mouse secondary antibody - A11001; Life Technologies, Eugene, USA), respectively. Prior to staining the cells were washed once in PBS, and thereafter permeabilized with T-PBS (0.1\% Triton X-100 in PBS; Sigma-Aldrich, St. Louis, USA) for 15 minutes. The T-PBS was removed and the cells were incubated for 2 hours in a $2 \%$ bovine seric albumin blocking agent to avoid unspecific binding. Monoclonal primary mouse anti-human vinculin antibodies (V9131, Sigma-Aldrich, St. Louis, USA) were added in excess for 1.5 hours. Finally, the cells were washed three times in T-PBS, incubated with DAPI, Phalloidin and Alexa Fluor 488 overnight, and thereafter washed twice with T-PBS. When not analyzed the cells were stored at $4^{\circ} \mathrm{C}$ in PBS.

Olympus cellSens dimension software was used to operate the Olympus BX 61 microscope (Olympus, Tokyo, Japan), and the nucleus and cytoskeleton of the cells adhered to the plastic coverslips were visualized. The "Count and Measure" function in the software Olympus cellSens dimension was used to quantify the PDLCs spreading area. An automatized threshold was set to $55 \mu \mathrm{m}^{2}$ of area, and the visibility of the nucleus was defined as what should be considered as a cell.

\section{Cell proliferation}

Cells were seeded at a density of $15 \times 10^{3}$ cells $/ \mathrm{cm}^{2}$ in 24-well plates, divided according to the groups, in the presence or absence of Sr. The cells were harvested after 1, 3, and 7 days of culture. For each period, the cells were trypsinized with $0.25 \%$ TrypLE $^{\text {TM }}$ Express reagent, no-phenol red (Gibco, Paisley, UK), and thereafter counted using NucleoCounter $^{\mathrm{TM}}$ (ChemoMetec, Allerod, Denmark). The cell count was made by adding $100 \mu \mathrm{L}$ of culture sample to $100 \mu \mathrm{L}$ of lysis buffer in a tube and was shaken vigorously. Later, $100 \mu \mathrm{L}$ of staining was added to the mixture and, again, shaken vigorously. Approximately $100 \mu \mathrm{L}$ of sample was loaded into the NucleoCassete ${ }^{\mathrm{TM}}$. This procedure 
provided a total cell count (cells $/ \mathrm{mL}$ ), based on the number of stained cell nuclei, in about $30 \mathrm{sec}$.

\section{Alkaline phosphatase activity assay}

The ability of alkaline phosphatase (ALP) to hydrolyse the colourless substrate pnitrophenyl phosphate (pNPP) (Invitrogen, Camarillo, USA) into a yellowish end-product, p-nitro-phenol (pNP) was used to quantify the ALP activity in the medium after 7 and 14 days of culture. PDLCs were seeded at $15 \times 10^{3}$ cells $/ \mathrm{cm}^{2}$ in 96 -well plates. Cells were washed with PBS and lysed with alkaline buffer containing $1.5 \mathrm{M}$ 2-amino-2-methyl-1propanol (100 $\mu \mathrm{L} /$ well; Sigma-Aldrich, St. Louis, USA), $\mathrm{pH} 10.3$, for 10 minutes at $37^{\circ} \mathrm{C}$. Then, $100 \mu \mathrm{L}$ pNPP substrate solution (1 mg/mL pNPP substrate in $0.1 \mathrm{M}$ Glycin buffer; Sigma-Aldrich, St. Louis, USA) was added to the cell lysate for 2.5 minutes at $37^{\circ} \mathrm{C}$, followed by addition of $100 \mu \mathrm{L} 2 \mathrm{M} \mathrm{NaOH}$ for 10 minutes to stop the enzymatic ALP conversion of pNPP into pNP. Spectrophotometrical quantification of pNP was performed on an EL800 absorbance microplate reader (BioTek, Winooski, USA) at a wavelength of $405 \mathrm{~nm}$. For a standard curve $1 \mathrm{mM}$ stock pNP was diluted from $0.05 \mathrm{mM}$ to $0.4 \mathrm{mM} \mathrm{pNP}$ (Sigma-Aldrich, St. Louis, USA) and used to calculate cell-specific ALP activity, which was expressed as $\mathrm{mM}$ pNP/minute/cell. The pNPP substrate and the $2 \mathrm{M} \mathrm{NaOH}$ stop solution were added at the same time as to the samples, providing the standard curve. Data were calculated for Sr and Ctrl groups after 7 and 14 days of culture.

\section{Gene expression - RNA isolation and real-time RT-PCR analysis}

PDLCs were investigated for their osteogenic gene expression after culture with Ctrl and Sr1-4. Real-time PCR (RT-PCR) was performed to evaluate the mRNA levels of osteogenesis-related genes alkaline phosphatase (ALP), runt-related transcription factor 2 (RUNX2), osteocalcin (OCN), osteopontin (OPN), and osteoprotegerin (OPG). For RT- 
PCR, PDLCs in Sr and Ctrl groups were seeded at $15 \times 10^{3}$ cells $/ \mathrm{cm}^{2}$ in 6 -well plates, in triplicate. After 1, 3, 7, 14, and 21 days of culture PDLCs were harvested and total RNA was extracted and purified using Macherey-Nagel total RNA kit (Macherey-Nagel, Düren, Germany) according to the manufacturer's instructions. RNA concentration and purity were spectrophotometrically determined using an Eppendorf BioPhotometer (Eppendorf, Hamburg, Germany) according to the manufacturer's instructions. The RNA samples were treated with recombinant DNase I (Macherey-Nagel, Düren, Germany) and converted into cDNA using cDNA synthesis kit (cat no 600559, Agilent Technologies, Santa Clara, USA). RT-PCR was performed on a Stratagene Mx3000P system (Stratagene, San Diego, USA) using TaqMan universal PCR master mix (Applied Biosystems, Waltham, USA) and TaqMan gene expression assays (Applied Biosystems, Waltham, USA) with the following primers: runt-related transcription factor 2 (RUNX2) Hs00231692_m1 (RUNX2), secreted phosphoprotein 1 (SPP1) Hs00959010_m1 (OPN), bone gamma-carboxyglutamate (gla) protein (BGLAP) Hs01587814_g1 (OCN), TNF receptor superfamily member 11b (TNFRSF11B) Hs 00900358_m1 (OPG), alkaline phosphatase-liver/bone/kidney (ALPL) Hs00758162_m1 (ALP) (Applied Biosystems, Waltham, USA). Standard enzyme and cycling conditions for the Stratagene Mx3000P system were used. Template cDNA corresponding to $1.176 \times 10^{-2} \mu \mathrm{g}$ of RNA was added to each PCR reaction and each biological sample was run in technical duplicates for each gene. Data analysis was performed using Stratagene Mx3000P real-time PCR system sequence detection software version 1.3 (Stratagene, San Diego, USA). Expression levels of the gene of interest were normalized to the "BestKeeper" index ${ }^{17}$, determined by the geometric mean of threshold cycles from ribosomal protein L13a, glucuronidase, beta and beta-2-microglobulin.

\section{Mineralization}


Differentiated osteoblasts can be induced to produce vast extracellular calcium deposits in vitro (i.e. mineralization) and these deposits can be stained in bright orange-red using Alizarin Red staining. ${ }^{18}$ To assess calcium deposition by Alizarin Red staining, PDLCs were seeded at $15 \times 10^{3}$ cells $/ \mathrm{cm}^{2}$ in 24 -well plates. After 14 and 21 days of culture, PDLCs were washed with PBS and fixed with $70 \%$ ethanol for at least 1 hour at $-20^{\circ} \mathrm{C}$. The fixed PDLCs were washed with double distilled water $\left(\mathrm{ddH}_{2} \mathrm{O}\right)$ and stained with $0.2 \%$ Alizarin Red (Sigma-Aldrich, St. Louis, USA) for 15 minutes, with rotation and at room temperature. Then the Alizarin Red solution was carefully aspirated and the cell monolayer was washed five times with $\mathrm{ddH}_{2} \mathrm{O}$ to remove non-specific staining. The cells were then air-dried. Images of the remaining red-orange spots, formed by the chromogenic complex between o-cresolphthaleon and calcium ions were captured at 10x magnification using a light microscope (Olympus IX73, Tokyo, Japan) and a digital camera (Olympus, Tokyo, Japan). For this experiment, an additional control group containing Sr4 in the medium, but with no cells was also tested, to allow the visualization of false positive results due to possible interaction between Sr in high concentrations and the Alizarin Red staining.

\section{Statistical analysis}

All experiments were performed in triplicate (i.e. in all experiments, three samples per group/period were tested). The data were described as means and standard deviations. GraphPad Prism 6.0 for Windows (GraphPad Software Inc., La Jolla, USA) was used for the statistical evaluation. Normality of the data was tested and confirmed with the Kolgomorov-Smirnov test, and comparisons (group- and period-wise) were made using Tukey post-hoc test followed a parametric one-way ANOVA. The minimum statistical significance was set at $p \leq 0.05$.

\section{Results}




\section{Cell morphology - spreading area}

Considering the spreading area of the cultured cells, Sr3 and Sr4 lead to larger areas than those associated to $\mathrm{Ctrl}, \mathrm{Sr} 1$ and $\mathrm{Sr} 2$ at 4 hours period. Ctrl, Sr1, and Sr2 groups reached the same areas only after 6 hours of evaluation. Yet, at 24 hours, Sr3 and Sr4 showed larger values than the control group ( $p \leq 0.05$ and $p \leq 0.01$, respectively). These results are presented in Figure 1.

\section{Cell proliferation}

As shown in Figure 2, for all Sr groups as well as the control, cell counts increased significantly according to the assessment period (i.e. from 1 to 3 , and then from 3 to 7 days of culture, $p \leq 0.001)$. Considering the differences among the groups within the same assessment period of evaluation, $\mathrm{Sr} 3$ and $\mathrm{Sr} 4$ showed significantly larger number of cells compared to lower Sr concentrations and the control group. At 7 days, Sr4 also led to a significantly larger number of cells than $\operatorname{Sr} 3(p \leq 0.001)$.

\section{ALP activity}

For all groups, there was a significant increase in ALP activity from 7 to 14 days $(p \leq 0.001)$. Sr4 showed significantly lower ALP activity at both 7 and 14 days when compared to Ctrl

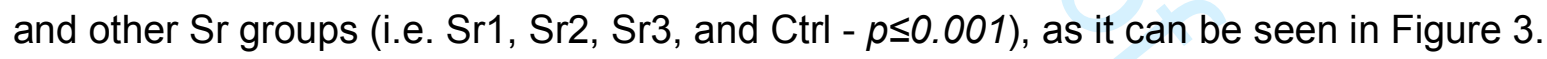

\section{Gene expression}

RT-PCR showed that osteogenic gene expression was altered by the addition of $\mathrm{Sr}$ to the culture medium, especially considering the highest tested $\mathrm{Sr}$ concentration (Sr4). Expression of RUNX2 was transiently lower at 7 and 14 days, when subject to Sr4, compared to the other groups. Overall, the peak of gene expression took place at 7 days. At 1, 3, and 21 days, all groups showed equal results regarding RUNX2 expression, but at 7 days, Sr4 showed lower levels of gene expression than all other groups (Figure 4). 
Considering ALP, and as seen in figure 5, the gene expression for $\mathrm{Sr} 4$ group was significantly lower than that seen for the other groups at $3,7,14$, and 21 days. In all of these evaluation periods, $\mathrm{Sr} 4$ lead to values that were several times lower than those found for the other $\mathrm{Sr}$ concentrations and the control group.

The expression patterns of the late osteoblastic differentiation markers OPN and OCN were both up-regulated in Sr4 (Figure 6 and 7, respectively). While for the control, Sr1, and Sr2 groups OPN expression decreased from 1 to 21 days, the Sr3 and Sr4 groups showed a diverse development, with higher levels of gene expression being found at 14 and 21 days when compared to day 1 (Figure 6). OCN expression showed a peak at 7 day for all groups, reaching lower values after that. Sr4 showed significantly larger gene expression compared to all other groups at 7, 14, and 21 days (Figure 7).

$\mathrm{Sr} 4 \mathrm{OPG}$ gene expression was up-regulated compared to the other Sr concentrations at 7 days. The other $\mathrm{Sr}$ concentrations, as well as the control group reached similar expression levels at 14 days of evaluation. However, at 21 days, Sr3 and Sr4 showed larger values than the other groups ( $\mathrm{Sr} 2, \mathrm{Sr} 1$, and $\mathrm{Ctrl})$. For both $\mathrm{Sr} 3$ and $\mathrm{Sr} 4$, the difference was with a significance of $p \leq 0.01$, compared to $\mathrm{Sr} 2$, $\mathrm{Sr} 1$, and Ctrl.These results are presented in Figure 8.

\section{Mineralization}

Mineralization, as assessed by subjective alizarin-red-positive nodules formed in the PDLC cultures after 14 and 21 days, was more evident and in larger quantity for the Sr4 group, when compared to the other groups, although some positive nodules were also seen for Sr3 (figure 9).

\section{Discussion}


One of the most important processes considering periodontal regeneration is inducing the proliferation and differentiation of the PDLCs into cells leading to de novo formation of the lost tissues. ${ }^{7}$ In the past decades, there have been many attempts in dental pharmacology and guided tissue regeneration to improve the condition for the remaining PDLCs in a compromised tooth in order for these cells to perform their function in the regeneration of PDL. ${ }^{16}$ Among the tissues which could be regenerated, bone is one of the most studied. As a result, the differentiation of PDLCs into osteoblast-like cells is a highly relevant feature in tissue engineering. ${ }^{9}$ In the present study, the choice of PDLCs was related to the osteoblast-like characteristics of this specific cell population. ${ }^{16}$ The literature suggests that roughly $30 \%$ of PDLCs retain the potential to differentiate into cells with osteoblastic properties. $^{7}$ To stimulate and enhance this differentiation process, several substances have been tested, ranging from bone-like materials (e.g. hydroxyapatite), ${ }^{19}$ to vitamins, ${ }^{20}$ polypeptides, and proteins (e.g. enamel matrix derivatives ${ }^{21}$ and bone morphogenetic proteins - BMPs). ${ }^{22} \mathrm{~A}$ recently-added candidate to be included in this test-list is $\mathrm{Sr}^{10,11}$ This addition is mostly related to the so called "dual action" of Sr: it can promote new bone formation by inducing the differentiation of pre-osteoblastic cells into osteoblasts ${ }^{23}$ and inhibit the formation and activation of bone resorbing cells (i.e. osteoclasts). ${ }^{24}$ Additionally, studies also refer to a possible antibacterial activity of Sr, which could be beneficial in the event of using this substance as an enhancer for regenerative procedures. ${ }^{25,26}$

In the present study the higher Sr concentration tested (Sr4) enhanced PDLCs growth and osteogenic behavior (i.e. gene expression and mineralization), considering all used assessment methodologies. This high concentration of $\mathrm{Sr}$ was selected to be comparable to that reported as possible in an actual in vivo system regarding local delivery of $\mathrm{Sr}^{10} \mathrm{~A}$ few other substances have been found to achieve similar results, mostly plateletderived growth factor (PDGF), platelet rich plasma (PRP), and BMPs. ${ }^{27-29}$ On the other hand, such substances have also been associated with some disadvantages such as high 
costs, high supra-physiological doses of growth factors, as well as unwanted side effects associated with recombinant therapies. ${ }^{30}$ Studies reporting on possible side effects of $\mathrm{Sr}$ administration (local and systemic) have no findings which would support such deleterious effects. ${ }^{10,31,32}$ In such studies, the tested Sr concentration was either based on what is relevant for the treatment of osteoporosis and bone pathologies (in the dose of $2 \mathrm{mg}$ of strontium ranelate per day), ${ }^{31,32}$ or being locally-delivered from implant surfaces, as an attempt to enhance the osseointegration of the implants. ${ }^{10}$ This goes toward the results of the only study found in the literature regarding the use of $\mathrm{Sr}$ for enhancing the proliferation of PDLCs, which also ratifies the promotion of PDLC proliferation following the use of high concentration of $\mathrm{Sr}^{16}$ In the referred study, it is found that $\mathrm{Sr}$ in the concentration of $3 \mathrm{mM}$ (or approximately $260 \mathrm{mg} / \mathrm{L}$ ) also lead to a significant increase in the cell count of a PDLC population. ${ }^{16}$ This concentration is intermediate to $\mathrm{Sr} 3$ and $\mathrm{Sr} 4$ as tested in the present study, and therefore ratifies the present findings.

Regarding the mechanisms that could be related to this effect on PDLC proliferation, there are several options described in the literature, which could be used to support the present findings. One of these mechanisms would be the interaction with the mitogen-activated protein kinase (MAPK) signaling pathway, which enhances the differentiation of mesenchymal stem cells into osteogenic cells. ${ }^{33}$ Further, $\mathrm{Sr}$ is known to induce prostaglandin production and cyclooxygenase expression in undifferentiated cells, thereby increasing their osteoblastic differentiation. ${ }^{34}$ Also, Sr might interact with fibroblast growth factor receptors present in the PDLCs, thereby promoting osteoblast synthetic activity. ${ }^{35}$ Another plausible mechanism would be the interaction with the cellular calciumsensing receptor (or another functionally different cation-sensing receptor) leading to its activation in the osteoblastic-cells, which would interfere in all phases of bone production, starting with cell differentiation and proliferation, therefore leading to enhanced bone matrix production. ${ }^{36}$ 
With the intent to gain more knowledge regarding these cellular mechanisms, in the present study we also evaluated the osteogenic behavior of PDLCs, by assessing ALP activity, and the expression of some relevant osteogenic genes (ALP, RUNX2, OPN, OCN, and OPG). We found that PDLCs cultured with $\mathrm{Sr} 4$ showed an increased gene expression of two important bone matrix proteins, OCN and OPN, from 7 days when compared to all other tested $\mathrm{Sr}$ concentrations $(\mathrm{Sr} 1, \mathrm{Sr}$, and $\mathrm{Sr} 3)$ and the control group. Moreover, lower ALP activity (from 7 days), and lower ALP gene expression (from 3 days) as well as a transiently lower RUNX2 gene expression (presenting statistical significance at 7 and 14 days) was also observed for cells subjected to Sr4, relative to the other groups. When subjected to Sr4, the peak for RUNX2 was found at 3 days. The data suggest that the Sr4 concentration positively influenced the differentiation and the maturation of the tested cell population into osteoblasts.

The RUNX2 and ALP gene expression results are also in agreement with an accelerated pathway for the differentiation of the PDLCs into osteogenic cells. One possible mechanism-based explanation for the present findings is that although RUNX2 triggers the expression of major bone matrix protein genes, it does not play a major role in the maintenance of the osteogenic gene expression profile in mature osteoblasts. ${ }^{37}$ During bone tissue formation, RUNX2 induces osteoblast differentiation and increases the number of cells forming immature bone, whereas RUNX2 expression has to be downregulated for differentiation into mature osteoblasts, which form mature bone. ${ }^{37}$ In other words, RUNX2 triggers the expression of major bone matrix genes during the early stages of osteoblast differentiation, but it is not essential for the maintenance of gene expression in mature osteoblast. ${ }^{38-40}$ Supporting the present results (i.e. low RUNX2 gene expression levels), studies focusing on the regulation of cell cycle progression suggest that RUNX2 expression increases when cell proliferation is slow, but it is markedly reduced during rapid cell proliferation. ${ }^{41,42}$ As for ALP, other studies with PDLCs point to the fact 
that, when cell proliferation is increased (as seen for Sr4), ALP gene expression will be downregulated. ${ }^{43,44}$

This is also in agreement with the significant up-regulation of OCN and OPN observed from 7 days. In relation to this, multiple mechanisms supporting the present results have been identified. The most prominent of these is the one suggesting that $\mathrm{Sr}$ would act in a somewhat similar fashion as BMP-2, through the activation of the Wnt/ $\mathrm{B}$ catenin pathway. ${ }^{45}$ In that direction, and similar to what is seen for BMP-2, Sr would interfere with the activation of the $\mathrm{Wnt} / \mathrm{\beta}$-catenin pathway, causing the translocation of transcription factor nuclear factor of activated T cells (NFAT) to the cell nucleus. ${ }^{45}$ This would upregulate $\beta$-catenin activation, affecting cell replication processes. Our findings regarding OCN and OPN expression also support the hypothesis regarding the interference of $\mathrm{Sr}$ in the $\mathrm{Wnt} / \beta$-catenin pathway. This is the case since both proteins are directly linked to the same early osteoblastic differentiation phases connected to ALP and RUNX2, as stated above. ${ }^{46}$ Further investigation is needed defining the mechanism(s) of action of the high Sr concentrations on the osteogenic behavior of PDLCs.

Concerning the potential action of $\mathrm{Sr}$ suppressing bone resorption, significant changes regarding OPG expression were also found to be associated with the highest $\mathrm{Sr}$ concentration ( $\mathrm{Sr} 4)$ at 7 days). The effect of $\mathrm{Sr}$ modulating (i.e. enhancing) OPG expression in osteoblasts is well documented in the literature. ${ }^{47,48}$ OPG is involved in the regulation of osteoclast activation, therefore interfering with bone resorption. ${ }^{46} \mathrm{OPG}$ is one of two known receptor proteins that can bind to the receptor activator of nuclear factor kB ligand (RANKL). The other receptor protein is receptor activator of NF-kB (RANK). Since RANKL can only bind to one receptor at a time, OPG and RANK compete with one another ${ }^{49}$ In this way, when RANKL is bound to OPG, it blocks these chemical signals and prevents the activation of osteoclasts, thus, OPG acts as a "decoy" receptor, reducing osteoclast activation. ${ }^{49}$ Supporting the present findings regarding OPG expression, other 
studies have suggested that $\mathrm{Sr}$ would down regulate RANKL expression in osteoblastic cells (i.e. up-regulation of OPG resulting in downregulation of RANKL). ${ }^{50}$ This would, in the end, result in a decreased number of active osteoclasts. ${ }^{51}$

The final outcome which could be altered within the bone formation pathway, i.e. the deposition of mineralized bone matrix, was also assessed by examining the effect of the tested Sr concentrations on the deposition of Ca-rich minerals. The observed increase in $\mathrm{OCN}$ expression also suggests that mineralization was ongoing, since $\mathrm{OCN}$ is secreted by osteoblastic cells and directly implicated in the bone mineralization process. ${ }^{46}$ The present results show that mineralization was more pronounced for the Sr4 group, when compared to Sr1-3 and Ctrl groups. This finding is in line with the available literature, in which numerous reports of the concentration-dependent effect of $\mathrm{Sr}$ in the mineralization of bone tissue are found. ${ }^{45,52,53}$ In the present study, such phenomenon could not be observed, since the tested Sr concentrations were not selected based on a gradual, linear increase. Further, mineralization was apparently altered in a significant manner only for the highest tested Sr concentration. Finally, and supporting the present findings, one should notice that the culture medium used in the present study was a regular culture medium, and not an osteogenic medium (e.g. Dulbecco's modified Eagle's medium - DMEM). This selection was done to avoid possible biases that would eventually lead to the differentiation of the PDLCs into osteoblasts enhanced by other issues rather than the presence of $\mathrm{Sr}$ in the medium. In the end, the only variable of this study was the diverse $\mathrm{Sr}$ concentrations, as initially suggested in the objectives.

In conclusion, PDLCs responded to a high concentration of $\mathrm{Sr}(\mathrm{Sr} 4,360 \mathrm{mg} / \mathrm{L})$ with increased cellular proliferation and osteogenic behavior (as assessed by osteogenic gene expression and mineralization) in vitro. Such findings could be further explored to enhance new bone formation within periodontal regenerative therapies. 


\section{Acknowledgements}

The authors acknowledge financial support from Innovation Fund Denmark through the project ASTI (1382-00053B).

\section{References}

1. Larsson L, Decker AM, Nibali L, Pilipchuk SP, Berglundh T, Giannobile WV. Regenerative Medicine for Periodontal and Peri-implant Diseases. J Dent Res. 2016;95: 255-266.

2. Wu YC, Lin LK, Song CJ, Su YX, Tu YK. Comparisons of periodontal regenerative therapies: A meta-analysis on the long-term efficacy. J Clin Periodontol. 2017;44: 511-519. 3. Cortellini P, Tonetti MS. Clinical concepts for regenerative therapy in intrabony defects. Periodontol 2000. 2015;68: 282-307.

4. Gay IC, Chen S, MacDougall M. Isolation and characterization of multipotent human periodontal ligament stem cells. Orthod Craniofac Res. 2007;10: 149-160.

5. Basdra EK, Komposch G. Osteoblast-like properties of human periodontal ligament cells: an in vitro analysis. Eur J Orthod. 1997;19: 615-621.

6. Lin Z, Rios HF, Cochran DL. Emerging regenerative approaches for periodontal reconstruction: a systematic review from the AAP Regeneration Workshop. $J$ Periodontol. 2015;86: S134-152.

7. Nagatomo K, Komaki M, Sekiya I, et al. Stem cell properties of human periodontal ligament cells. J Periodontal Res. 2006;41: 303-310.

8. Ivanovski S, Li H, Haase HR, Bartold PM. Expression of bone associated macromolecules by gingival and periodontal ligament fibroblasts. J Periodontal Res. 2001;36: 131-141. 
9. Nakashima M, Reddi $\mathrm{AH}$. The application of bone morphogenetic proteins to dental tissue engineering. Nat Biotechnol. 2003;21: 1025-1032.

10. Andersen OZ, Offermanns V, Sillassen M, et al. Accelerated bone ingrowth by local delivery of strontium from surface functionalized titanium implants. Biomaterials. 2013;34: 5883-5890.

11. Park JW, Kim YJ, Jang JH, Song H. Positive modulation of osteogenesis- and osteoclastogenesis-related gene expression with strontium-containing microstructured $\mathrm{Ti}$ implants in rabbit cancellous bone. J Biomed Mater Res A. 2013;101: 298-306.

12. Burlet N, Reginster JY. Strontium ranelate: the first dual acting treatment for postmenopausal osteoporosis. Clin Orthop Relat Res. 2006;443: 55-60.

13. Li Y, Li X, Song G, Chen K, Yin G, Hu J. Effects of strontium ranelate on osseointegration of titanium implant in osteoporotic rats. Clin Oral Implants Res. 2012;23: 1038-1044.

14. Maimoun L, Brennan TC, Badoud I, Dubois-Ferriere V, Rizzoli R, Ammann P. Strontium ranelate improves implant osseointegration. Bone. 2010;46: 1436-1441.

15. Er K, Polat ZA, Ozan F, Tasdemir T, Sezer U, Siso SH. Cytotoxicity analysis of strontium ranelate on cultured human periodontal ligament fibroblasts: a preliminary report. J Formos Med Assoc. 2008;107: 609-615.

16. Romer P, Desaga B, Proff P, Faltermeier A, Reicheneder C. Strontium promotes cell proliferation and suppresses IL-6 expression in human PDL cells. Ann Anat. 2012;194: 208-211.

17. Pfaffl MW, Tichopad A, Prgomet C, Neuvians TP. Determination of stable housekeeping genes, differentially regulated target genes and sample integrity: BestKeeper--Excel-based tool using pair-wise correlations. Biotechnology letters. 2004;26: 509-515. 
18. Stanford CM, Jacobson PA, Eanes ED, Lembke LA, Midura RJ. Rapidly forming apatitic mineral in an osteoblastic cell line (UMR 106-01 BSP). J Biol Chem. 1995;270: 9420-9428.

19. Kao RT, Nares S, Reynolds MA. Periodontal regeneration - intrabony defects: a systematic review from the AAP Regeneration Workshop. J Periodontol. 2015;86: S77104.

20. Stein SH, Livada R, Tipton DA. Re-evaluating the role of vitamin D in the periodontium. J Periodontal Res. 2014;49: 545-553.

21. Miron RJ, Dard M, Weinreb M. Enamel matrix derivative, inflammation and soft tissue wound healing. J Periodontal Res. 2015;50: 555-569.

22. Carreira AC, Lojudice FH, Halcsik E, Navarro RD, Sogayar MC, Granjeiro JM. Bone morphogenetic proteins: facts, challenges, and future perspectives. J Dent Res. 2014;93: $335-345$.

23. Yang F, Yang D, Tu J, Zheng Q, Cai L, Wang L. Strontium enhances osteogenic differentiation of mesenchymal stem cells and in vivo bone formation by activating Wnt/catenin signaling. Stem Cells. 2011;29: 981-991.

24. Peng S, Liu XS, Huang S, et al. The cross-talk between osteoclasts and osteoblasts in response to strontium treatment: involvement of osteoprotegerin. Bone. 2011;49: 12901298.

25. He G, Wu Y, Zhang Y, et al. Addition of Zn to the ternary Mg-Ca-Sr alloys significantly improves their antibacterial property. J Mater Chem B Mater Biol Med. 2015;3: 6676-6689.

26. Brauer DS, Karpukhina N, Kedia G, et al. Bactericidal strontium-releasing injectable bone cements based on bioactive glasses. J R Soc Interface. 2013;10: 20120647.

27. Kobayashi E, Fluckiger L, Fujioka-Kobayashi M, et al. Comparative release of growth factors from PRP, PRF, and advanced-PRF. Clin Oral Investig. 2016;20: 2353-2360. 
28. Kobayashi E, Fujioka-Kobayashi M, Sculean A, et al. Effects of platelet rich plasma (PRP) on human gingival fibroblast, osteoblast and periodontal ligament cell behaviour. BMC Oral Health. 2017;17: 91.

29. Miron RJ, Zhang YF. Osteoinduction: a review of old concepts with new standards. J Dent Res. 2012;91: 736-744.

30. Rocque BG, Kelly MP, Miller JH, Li Y, Anderson PA. Bone morphogenetic proteinassociated complications in pediatric spinal fusion in the early postoperative period: an analysis of 4658 patients and review of the literature. J Neurosurg Pediatr. 2014;14: 635643.

31. Meunier PJ, Roux C, Ortolani S, et al. Effects of long-term strontium ranelate treatment on vertebral fracture risk in postmenopausal women with osteoporosis. Osteoporos Int. 2009;20: 1663-1673.

32. Reginster JY, Seeman E, De Vernejoul MC, et al. Strontium ranelate reduces the risk of nonvertebral fractures in postmenopausal women with osteoporosis: Treatment of Peripheral Osteoporosis (TROPOS) study. J Clin Endocrinol Metab. 2005;90: 2816-2822.

33. Peng S, Zhou G, Luk KD, et al. Strontium promotes osteogenic differentiation of mesenchymal stem cells through the Ras/MAPK signaling pathway. Cell Physiol Biochem. 2009;23: 165-174.

34. Choudhary S, Halbout $P$, Alander C, Raisz L, Pilbeam C. Strontium ranelate promotes osteoblastic differentiation and mineralization of murine bone marrow stromal cells: involvement of prostaglandins. J Bone Miner Res. 2007;22: 1002-1010.

35. Caverzasio J, Thouverey C. Activation of FGF receptors is a new mechanism by which strontium ranelate induces osteoblastic cell growth. Cell Physiol Biochem. 2011;27: 243250.

36. Pors Nielsen S. The biological role of strontium. Bone. 2004;35: 583-588. 
37. Komori T. Regulation of bone development and extracellular matrix protein genes by RUNX2. Cell and tissue research. 2010;339: 189-195.

38. Komori T. Regulation of osteoblast differentiation by Runx2. Adv Exp Med Biol. 2010;658: 43-49.

39. Inada M, Yasui T, Nomura S, et al. Maturational disturbance of chondrocytes in Cbfa1deficient mice. Dev Dynam. 1999;214: 279-290.

40. Lee MH, Javed A, Kim HJ, et al. Transient upregulation of CBFA1 in response to bone morphogenetic protein-2 and transforming growth factor beta1 in $\mathrm{C} 2 \mathrm{C} 12$ myogenic cells coincides with suppression of the myogenic phenotype but is not sufficient for osteoblast differentiation. J Cel Biochem. 1999;73: 114-125.

41. Galindo M, Kahler RA, Teplyuk NM, et al. Cell cycle related modulations in Runx2 protein levels are independent of lymphocyte enhancer-binding factor 1 (Lef1) in proliferating osteoblasts. J Mol Hist. 2007;38: 501-506.

42. Xu J, Li Z, Hou Y, Fang W. Potential mechanisms underlying the Runx2 induced osteogenesis of bone marrow mesenchymal stem cells. Am J Transl Res. 2015;7: 25272535.

43. Choi MH, Noh WC, Park JW, Lee JM, Suh JY. Gene expression pattern during osteogenic differentiation of human periodontal ligament cells in vitro. $J$ Periodontal Implant Sci. 2011;41: 167-175.

44. Stein GS, Lian JB. Molecular mechanisms mediating proliferation/differentiation interrelationships during progressive development of the osteoblast phenotype. Endocr Rev. 1993;14: 424-442.

45. Zhang $\mathrm{W}$, Tian $\mathrm{Y}, \mathrm{He} \mathrm{H}$, et al. Strontium attenuates rhBMP-2-induced osteogenic differentiation via formation of Sr-rhBMP-2 complex and suppression of Smad-dependent signaling pathway. Acta Biomaterialia. 2016;33: 290-300. 
46. Chapurlat RD, Confavreux CB. Novel biological markers of bone: from bone metabolism to bone physiology. Rheumatology (Oxford). 2016;55: 1714-1725.

47. Tao ZS, Zhou WS, He XW, et al. A comparative study of zinc, magnesium, strontiumincorporated hydroxyapatite-coated titanium implants for osseointegration of osteopenic rats. Mat Science Engineer C, Mat Biol Appl. 2016;62: 226-232.

48. Yang HW, Lin MH, Xu YZ, Shang GW, Wang RR, Chen K. Osteogenesis of bone marrow mesenchymal stem cells on strontium-substituted nano-hydroxyapatite coated roughened titanium surfaces. Int J Clin Exp Med. 2015;8: 257-264.

49. Liu W, Zhang X. Receptor activator of nuclear factor-kappaB ligand (RANKL)/RANK/osteoprotegerin system in bone and other tissues (review). Mol Med Rep. 2015;11: 3212-3218.

50. Saidak Z, Marie PJ. Strontium signaling: Molecular mechanisms and therapeutic implications in osteoporosis. Pharmacology \& Therapeutics. 2012;136: 216-226.

51. Boyce BF, Xing L. The RANKL/RANK/OPG pathway. Curr Osteoporos Rep. 2007;5: 98-104.

52. Lindahl C, Pujari-Palmer S, Hoess A, Ott M, Engqvist $H$, Xia W. The influence of Sr content in calcium phosphate coatings. Mat Science Engineer C, Mat Biol Appl. 2015;53: 322-330.

53. Grynpas MD, Marie PJ. Effects of low doses of strontium on bone quality and quantity in rats. Bone. 1990;11: 313-319. 


\section{Figure legends}

Figure 1 PDLCs spreading area (mean \pm SD) for the control and the diverse $\mathrm{Sr}$ concentrations at the four evaluation periods. Ctrl, control group without $\mathrm{Sr} ; \mathrm{Sr} 1,11.3 \mathrm{x}$ $10^{-3} \mathrm{mg} / \mathrm{L}$ of $\mathrm{Sr} ; \mathrm{Sr} 2,13 \mathrm{mg} / \mathrm{L}$ of $\mathrm{Sr} ; \mathrm{Sr} 3,130 \mathrm{mg} / \mathrm{L}$ of $\mathrm{Sr} ; \mathrm{Sr} 4,360 \mathrm{mg} / \mathrm{L}$ of $\mathrm{Sr}$. The letters on top of each bar indicate statistical difference among the groups within the same period of evaluation. a - difference from Ctrl, $p \leq 0.05$; aa - difference from Ctrl, $p \leq 0.01$; aaa - difference from Ctrl, $p \leq 0.001 ; b$ - difference from $\mathrm{Sr} 1, p \leq 0.05 ; \mathrm{cc}$ - difference from Sr2, $p \leq 0.01$; ccc - difference from Sr2, $p \leq 0.001$; Tukey post hoc test followed ANOVA.

Figure 2 PDLCs proliferation (mean $\pm \mathrm{SD}$ ) for the control and the diverse $\mathrm{Sr}$ concentrations at the three evaluation periods. The letters on top of each bar indicate statistical difference among the groups within the same period of evaluation. aa difference from Ctrl, $p \leq 0.01$; aaa - difference from Ctrl, $p \leq 0.001 ; b$ - difference from Sr1, $p \leq 0.05$; bb - difference from Sr1, $p \leq 0.01$; bbb - difference from Sr1, $p \leq 0.001 ; c$ difference from $\mathrm{Sr} 2, p \leq 0.05$; $c c$ - difference from $\mathrm{Sr} 2, p \leq 0.01$; $c c c$ - difference from $\mathrm{Sr}$, $p \leq 0.001$; ddd - difference from Sr3, $p \leq 0.001$; Tukey post hoc test followed ANOVA.

Figure 3 ALP activity of the PDLCs (mean \pm SD) for the control and the diverse $\mathrm{Sr}$ concentrations at the two evaluation periods. The letters on top of each bar indicate statistical difference among the groups within the same period of evaluation. aaa difference from Ctrl, $p \leq 0.001$; bbb - difference from Sr1, $p \leq 0.001$; ccc - difference from Sr2, $p \leq 0.001$; ddd - difference from Sr3, $p \leq 0.001$; Tukey post hoc test followed ANOVA. 
Figure 4 RUNX2 gene expression of the PDLCs (mean \pm SD), assessed by RT-PCR. The letters on top of each bar indicate statistical difference among the groups within the same period of evaluation. a - difference from Ctrl, $p \leq 0.05$; aa - difference from Ctrl, $p \leq 0.01$; aaa - difference from Ctrl, $p \leq 0.001 ; b$ - difference from Sr1, $p \leq 0.05$; bbb difference from $\mathrm{Sr} 1, p \leq 0.001 ; \mathrm{c}$ - difference from $\mathrm{Sr} 2, p \leq 0.05 ; \mathrm{cc}$ - difference from $\mathrm{Sr}$, $p \leq 0.01$; ccc - difference from Sr2, $p \leq 0.001$; ddd - difference from Sr3, $p \leq 0.001$; Tukey post hoc test followed ANOVA.

Figure 5 ALP gene expression of the PDLCs (mean \pm SD), assessed by RT-PCR. The letters on top of each bar indicate statistical difference among the groups within the same period of evaluation. a - difference from Ctrl, $p \leq 0.05$; aa - difference from Ctrl, $p \leq 0.01 ;$ aaa - difference from Ctrl, $p \leq 0.001 ; b$ - difference from Sr1, $p \leq 0.05 ; b b$ difference from Sr1, $p \leq 0.01$; bbb - difference from Sr1, $p \leq 0.001$; ccc - difference from Sr2, $p \leq 0.001$; ddd - difference from Sr3, $p \leq 0.001$; Tukey post hoc test followed ANOVA.

Figure 6 OPN gene expression of the PDLCs (mean \pm SD), assessed by RT-PCR. The letters on top of each bar indicate statistical difference among the groups within the same period of evaluation. a - difference from Ctrl, $p \leq 0.05$; aaa - difference from Ctrl, $p \leq 0.001 ; b$ - difference from Sr1, $p \leq 0.05 ; b b b$ - difference from Sr1, $p \leq 0.001 ; c$ difference from Sr2, $p \leq 0.05$; ccc - difference from Sr2, $p \leq 0.001$; ddd - difference from $\mathrm{Sr} 3, p \leq 0.001$; Tukey post hoc test followed ANOVA. 
Figure 7 OCN gene expression of the PDLCs (mean \pm SD), assessed by RT-PCR. The letters on top of each bar indicate statistical difference among the groups within the same period of evaluation. aaa - difference from Ctrl, $p \leq 0.001 ; b$ - difference from Sr1, $p \leq 0.05$; bbb - difference from Sr1, $p \leq 0.001$; ccc - difference from Sr2, $p \leq 0.001$; ddd difference from Sr3, $p \leq 0.001$; Tukey post hoc test followed ANOVA.

Figure 8 OPG gene expression of the PDLCs (mean \pm SD), assessed by RT-PCR. The letters on top of each bar indicate statistical difference among the groups within the same period of evaluation. a - difference from Ctrl, $p \leq 0.05$; aa - difference from Ctrl, $p \leq 0.01$; aaa - difference from Ctrl, $p \leq 0.001 ; b$ - difference from Sr1, $p \leq 0.05$; bb difference from $\mathrm{Sr} 1, p \leq 0.01$; bbb - difference from Sr1, $p \leq 0.001$; c - difference from Sr2, $p \leq 0.05$; ; cc - difference from Sr2, $p \leq 0.01$; ccc - difference from Sr2, $p \leq 0.001$; d difference from Sr3, $p \leq 0.05$; Tukey post hoc test followed ANOVA.

Figure 9 Representative images of the Alizarin Red staining for Ca deposits (bone nodules) for the diverse $\mathrm{Sr}$ concentrations at the two evaluation periods. Magnification rate, 10x. A control group containing Sr4, but with no cells was also tested (Ctrl/Sr4), to allow the visualization of false positive results due to possible interaction between $\mathrm{Sr}$ in high concentrations and the Alizarin Red staining. The scale bars in the lower left corner of each image represents $100 \mu \mathrm{m}$. 
PDLCs spreading area (mean \pm SD) for the control and the diverse Sr concentrations at the four evaluation periods. Ctrl, control group without Sr; Sr1, $11.3 \times 10-3 \mathrm{mg} / \mathrm{L}$ of Sr; Sr2, $13 \mathrm{mg} / \mathrm{L}$ of Sr; Sr3, $130 \mathrm{mg} / \mathrm{L}$ of $\mathrm{Sr} ; \mathrm{Sr} 4,360 \mathrm{mg} / \mathrm{L}$ of Sr. The letters on top of each bar indicate statistical difference among the groups within the same period of evaluation. a - difference from Ctrl, $p \leq 0.05$; aa - difference from Ctrl, $p \leq 0.01$; aaa - difference from Ctrl, $\mathrm{p} \leq 0.001 ; \mathrm{b}$ - difference from Sr1, $\mathrm{p} \leq 0.05$; cc - difference from Sr2, $\mathrm{p} \leq 0.01$; ccc difference from $\mathrm{Sr} 2, \mathrm{p} \leq 0.001$; Tukey post hoc test followed ANOVA.

$187 \times 77 \mathrm{~mm}(300 \times 300 \mathrm{DPI})$ 


\section{Cell proliferation}

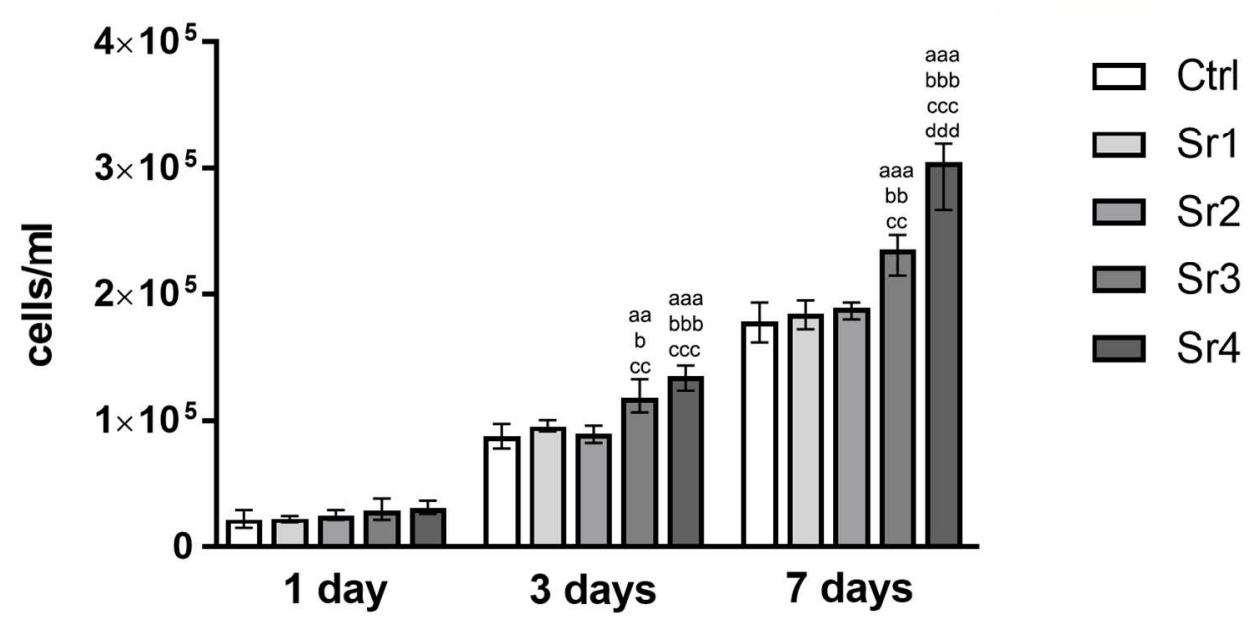

PDLCs proliferation (mean \pm SD) for the control and the diverse Sr concentrations at the three evaluation periods. The letters on top of each bar indicate statistical difference among the groups within the same period of evaluation. aa - difference from Ctrl, $p \leq 0.01$; aaa - difference from Ctrl, $p \leq 0.001$; $b$ - difference from Sr1, $p \leq 0.05 ; b b$ - difference from Sr1, $p \leq 0.01 ; b b b$ - difference from Sr1, $p \leq 0.001 ; c$ - difference from Sr2, $p \leq 0.05$; cc - difference from Sr2, $p \leq 0.01$; ccc - difference from Sr2, $p \leq 0.001$; ddd - difference from Sr3, $\mathrm{p} \leq 0.001$; Tukey post hoc test followed ANOVA.

$130 \times 77 \mathrm{~mm}(300 \times 300 \mathrm{DPI})$ 
ALP activity of the PDLCs (mean \pm SD) for the control and the diverse Sr concentrations at the two evaluation periods. The letters on top of each bar indicate statistical difference among the groups within the same period of evaluation. aaa - difference from Ctrl, $\mathrm{p} \leq 0.001$; bbb - difference from Sr1, $\mathrm{p} \leq 0.001$; ccc difference from Sr2, $\mathrm{p} \leq 0.001$; ddd - difference from Sr3, $\mathrm{s} \leq 0.001$; Tukey post hoc test followed ANOVA.

$160 \times 77 \mathrm{~mm}(300 \times 300 \mathrm{DPI})$ 
RUNX2 gene expression of the PDLCs (mean \pm SD), assessed by RT-PCR. The letters on top of each bar indicate statistical difference among the groups within the same period of evaluation. a - difference from Ctrl, $\mathrm{p} \leq 0.05$; aa - difference from Ctrl, $\mathrm{p} \leq 0.01$; aaa - difference from Ctrl, $\mathrm{p} \leq 0.001$; $\mathrm{b}$ - difference from $\mathrm{Sr} 1, \mathrm{p} \leq 0.05 ; \mathrm{bbb}$ - difference from Sr1, $\mathrm{p} \leq 0.001 ; \mathrm{c}$ - difference from Sr2, $\mathrm{p} \leq 0.05 ; \mathrm{cc}$ - difference from Sr2, $\mathrm{p} \leq 0.01$; ccc - difference from Sr2, $\mathrm{p} \leq 0.001$; ddd - difference from Sr3, $\mathrm{p} \leq 0.001$; Tukey post hoc test followed ANOVA.

$152 \times 82 \mathrm{~mm}(300 \times 300$ DPI $)$ 
ALP gene expression of the PDLCs (mean \pm SD), assessed by RT-PCR. The letters on top of each bar indicate statistical difference among the groups within the same period of evaluation. a - difference from Ctrl, $\mathrm{p} \leq 0.05$; aa - difference from Ctrl, $\mathrm{p} \leq 0.01$; aaa - difference from Ctrl, $\mathrm{p} \leq 0.001 ; \mathrm{b}$ - difference from Sr1, $\mathrm{p} \leq 0.05 ; \mathrm{bb}$ - difference from Sr1, $\mathrm{p} \leq 0.01 ; \mathrm{bbb}$ - difference from Sr1, $\mathrm{p} \leq 0.001$; ccc - difference from Sr2, $\mathrm{p} \leq 0.001$; ddd - difference from Sr3, $\mathrm{p} \leq 0.001$; Tukey post hoc test followed ANOVA.

$154 \times 82 \mathrm{~mm}(300 \times 300$ DPI $)$ 
OPN

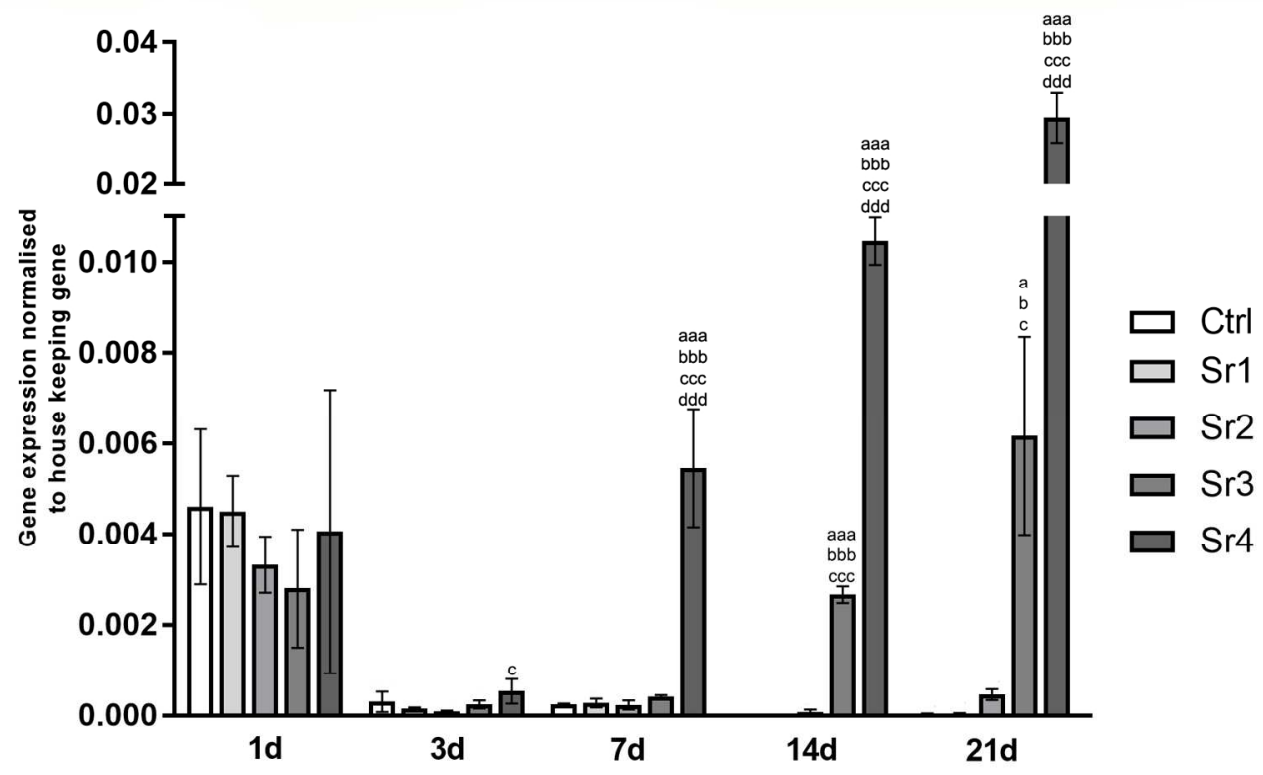

OPN gene expression of the PDLCs (mean \pm SD), assessed by RT-PCR. The letters on top of each bar indicate statistical difference among the groups within the same period of evaluation. a - difference from Ctrl, $\mathrm{p} \leq 0.05$; aaa - difference from Ctrl, $\mathrm{p} \leq 0.001 ; \mathrm{b}$ - difference from Sr1, $\mathrm{p} \leq 0.05$; bbb - difference from Sr1, $\mathrm{p} \leq 0.001$; $\mathrm{c}$ - difference from Sr2, $\mathrm{p} \leq 0.05$; ccc - difference from Sr2, $\mathrm{p} \leq 0.001$; ddd - difference from Sr3, $\mathrm{p} \leq 0.001$; Tukey post hoc test followed ANOVA.

$156 \times 108 \mathrm{~mm}(300 \times 300 \mathrm{DPI})$ 
OCN gene expression of the PDLCs (mean \pm SD), assessed by RT-PCR. The letters on top of each bar indicate statistical difference among the groups within the same period of evaluation. aaa - difference from Ctrl, $\mathrm{p} \leq 0.001$; $\mathrm{b}$ - difference from Sr1, p $\leq 0.05$; bbb - difference from Sr1, $\mathrm{p} \leq 0.001$; ccc - difference from Sr2, $\mathrm{p} \leq 0.001$; ddd - difference from Sr3, $\mathrm{p} \leq 0.001$; Tukey post hoc test followed ANOVA.

$156 \times 87 \mathrm{~mm}(300 \times 300$ DPI $)$ 


\section{OPG}

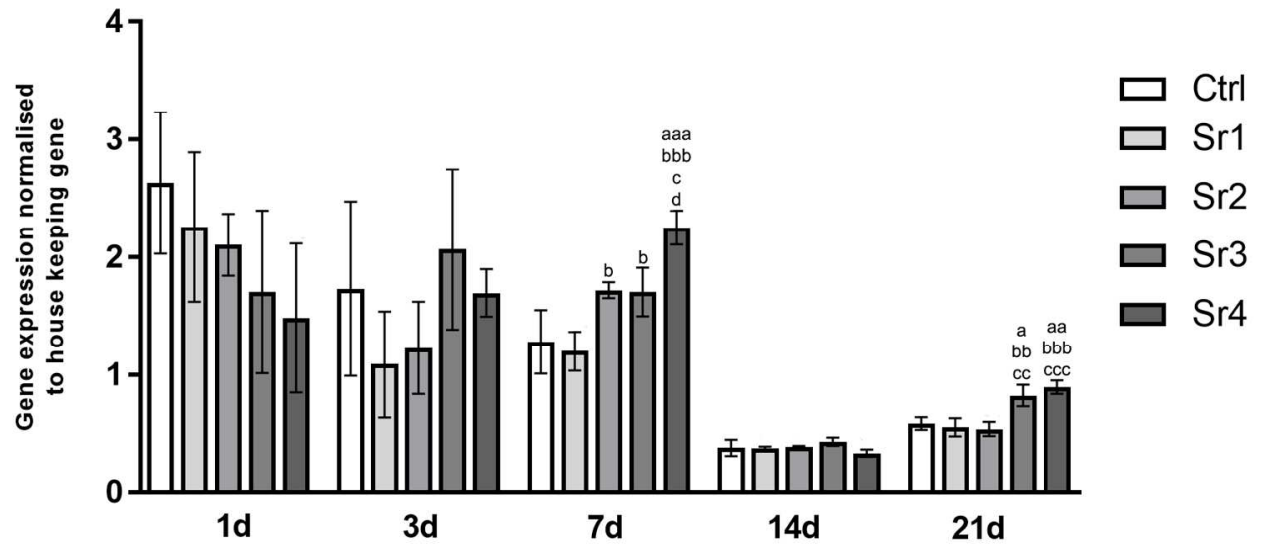

OPG gene expression of the PDLCs (mean \pm SD), assessed by RT-PCR. The letters on top of each bar indicate statistical difference among the groups within the same period of evaluation. a - difference from Ctrl, $\mathrm{p} \leq 0.05$; aa - difference from Ctrl, $\mathrm{p} \leq 0.01$; aaa - difference from Ctrl, $\mathrm{p} \leq 0.001 ; \mathrm{b}$ - difference from Sr1, $p \leq 0.05 ; b b$ - difference from Sr1, $p \leq 0.01 ; b b b$ - difference from Sr1, $p \leq 0.001 ; c$ - difference from Sr2, $\mathrm{p} \leq 0.05$; $; \mathrm{cc}$ - difference from Sr2, $\mathrm{p} \leq 0.01$; ccc - difference from Sr2, $\mathrm{p} \leq 0.001 ; \mathrm{d}$ - difference from $\mathrm{Sr}$, $\mathrm{p} \leq 0.05$; Tukey post hoc test followed ANOVA.

$152 \times 82 \mathrm{~mm}(300 \times 300$ DPI $)$ 


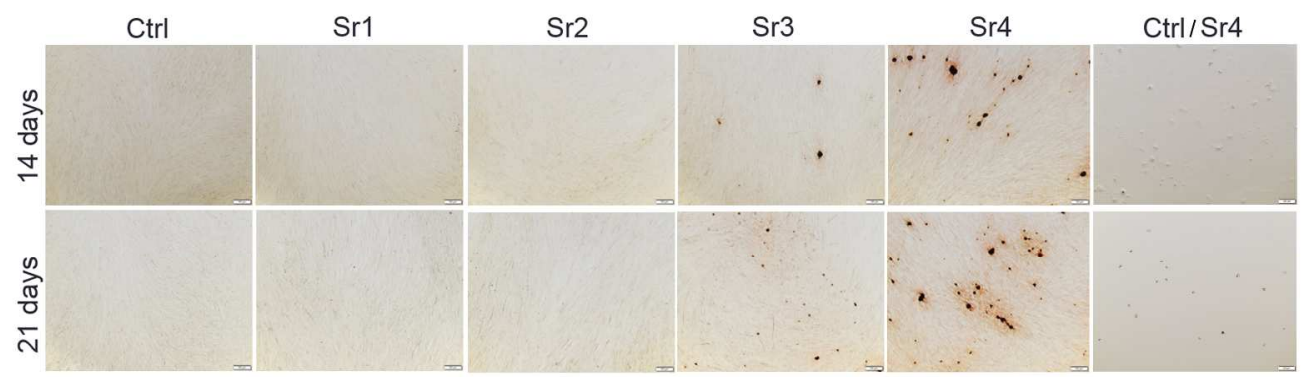

Representative images of the Alizarin Red staining for Ca deposits (bone nodules) for the diverse $\mathrm{Sr}$ concentrations at the two evaluation periods. Magnification rate, 10x. A control group containing Sr4, but with no cells was also tested (Ctrl/Sr4), to allow the visualization of false positive results due to possible interaction between $\mathrm{Sr}$ in high concentrations and the Alizarin Red staining. The scale bars in the lower left corner of each image represents $100 \mu \mathrm{m}$.

\section{$237 \times 68 \mathrm{~mm}(300 \times 300 \mathrm{DPI})$}

